\title{
Ritmi circadiani e variabili metaboliche
}

\author{
Sara Benazzi ${ }^{1} \cdot$ Stefania Gorini $^{2} \cdot$ Alessandra Feraco $^{1,2} \cdot$ Massimiliano Caprio $^{1,2}$
}

Accettato: 15 settembre 2021 / Pubblicato online: 5 novembre 2021

(c) The Author(s), under exclusive licence to Springer Nature Switzerland AG 2021

Sommario I ritmi circadiani sono influenzati da numerose variabili correlate allo stile di vita, soprattutto in relazione ai ritmi imposti dalla società moderna, e vengono profondamente alterati da diverse condizioni patologiche. La fisiologia circadiana è organizzata in modo complesso e integrato; molti dei fattori che sincronizzano il sistema sono a loro volta influenzati e regolati da diversi assi ormonali. Parallelamente, i disturbi del ritmo circadiano derivano da input non ottimali dei fattori sincronizzanti o da condizioni patologiche, e le conseguenze determinano un impatto significativo in diverse condizioni, quali l'obesità e i disturbi del sonno. Durante l'attuale emergenza COVID-19 sono stati registrati crescenti tassi di alterazioni del sonno, complici la preoccupazione diffusa, un comportamento alimentare alterato e la difficoltà per molti, durante il lockdown, nel mantenere ritmi di vita regolari (Barrea et al. in J Transl Med 18:1-11, 2020). Le misure di intervento che si sono mostrate più promettenti contro la desincronizzazione circadiana sono quelle che agiscono sullo stile di vita, basate sul recupero di un corretto ritmo del sonno, la corretta esposizione alla luce solare, l'idonea distribuzione dei pasti e del timing alimentare e lo svolgimento di un'adeguata attività fisica.

Proposto da Massimiliano Caprio.

Informazioni Supplementari La versione online contiene materiale supplementare disponibile su https://doi.org/10.1007/s40619-021-00983-5.

$凶$ M. Caprio

massimiliano.caprio@sanraffaele.it

1 Dipartimento di Scienze Umane e Promozione della Qualità della Vita, Università San Raffaele Roma, Roma, Italia

2 Laboratorio di Endocrinologia Cardiovascolare, IRCCS San Raffaele Roma, Roma, Italia
Parole chiave Ritmi circadiani - Desincronizzazione . Timing alimentare

\section{Introduzione}

I ritmi circadiani sono determinati da oscillazioni di parametri fisiologici e metabolici nell' arco delle 24 ore; il termine circadiano significa "intorno al giorno". Il sistema circadiano anticipa i cambiamenti ambientali, per ottimizzare l'adattamento dell'organismo in base al momento della giornata, determinando una coordinata segregazione temporale di numerosi processi biochimici, al fine di renderli maggiormente efficienti. Le ricerche che hanno portato alla scoperta dei meccanismi molecolari che controllano i ritmi circadiani sono valse il premio Nobel nel 2017 a Jeffrey C. Hall, Michael Rosbash e Michael W. Young.

Il sistema si compone di orologi (clocks) circadiani, oscillatori biologici localizzati in specifiche aree del sistema nervoso centrale o in tessuti periferici. L'organizzazione dei clocks è gerarchica: all'apice troviamo il master clock, localizzato nel nucleo soprachiasmatico (SCN) dell'ipotalamo, controllato principalmente dai segnali luminosi. Al di sotto troviamo clocks encefalici secondari e clocks periferici, distribuiti in vari organi e tessuti, maggiormente sensibili a segnali di tipo metabolico e nutrizionale [2]. È stata dimostrata l'attività ciclica spontanea dei neuroni del SCN, sostenuta da un sistema integrato di feedback trascrizionali e post-traduzionali.

Il meccanismo molecolare circadiano consiste in un loop trascrizionale che coinvolge diversi geni. I geni Clock e Bmal1 codificano per le proteine CLOCK e BMAL1, appartenenti al gruppo di proteine note come basic Helix-LoopHelix Per-Arnt-Single-minded (bHLH-PAS), che rappresentano la parte positiva del circuito di feedback. CLOCK e 


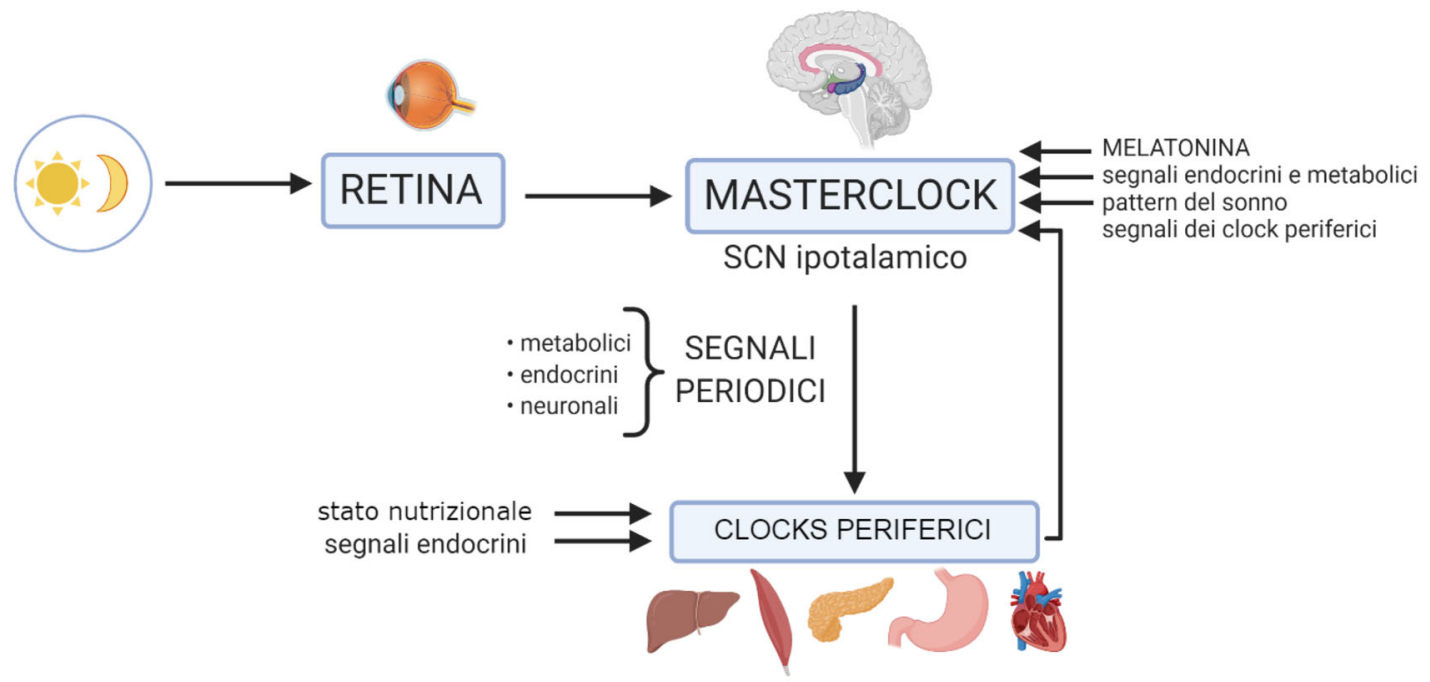

Fig. 1 Rappresentazione schematica della regolazione integrata del sistema gerarchico circadiano

BMAL1 formano un eterodimero che induce la trascrizione dei geni target, legandosi a specifici siti di DNA nei loro promotori. I geni attivati includono le componenti negative del loop: i geni Per1, Per2, Cry1 e Cry2. Le proteine codificate da tali geni, PER e CRY, dimerizzano e bloccano l'attività trascrizionale di CLOCK:BMAL1 [3]. Questo programma di espressione genica viene controllato anche grazie a eventi ciclici di rimodellamento della cromatina e di controllo epigenetico. La proteina CLOCK possiede un dominio hystone acetyl transferase (HAT) e la deacetilasi SIRT1, che è coinvolta nella deacetilazione istonica nei promotori di alcuni geni circadiani, è sensibile alle concentrazioni cellulari di NAD+, evidenziando quanto anche il metabolismo cellulare influisca sulla regolazione della trascrizione dei geni del clock [3].

Il dimero CLOCK:BMAL1 induce anche la trascrizione di elementi di un secondo loop, che agisce in concerto con il primo e coinvolge la trascrizione dei geni Rev-Erb $\alpha / \beta$ e $\operatorname{ROR} \alpha / \beta$ mediante legame a E-box. Le proteine tradotte da tali geni denominate REV-ERB e ROR legano siti del promotore di Bmall, inducendone e inibendone, rispettivamente, la trascrizione [3].

L'oscillazione circadiana è regolata in primis dal ciclo luce-buio, tramite il fotopigmento melanopsina, prodotto dalle cellule dei gangli della retina. La regolazione è finemente controllata anche da input metabolici e comportamentali, come lo stato nutrizionale e il ritmo sonno-veglia. Questi meccanismi vengono rinforzati anche da numerosi segnali biochimici ed elettrici ausiliari, come il loop RORalpha REV-ERBalpha, interazioni glia-neuroni e impulsi elettrici a livello delle cellule del SCN. Gli ouptut circadiani centrali a loro volta regolano i pattern fisiologici e comportamentali e forniscono segnali per la sincro- nizzazione dei clocks periferici, in un sistema integrato e interconnesso (Fig. 1) [4].

\section{La fisiologia dei ritmi circadiani}

\section{Sincronizzatori}

I sincronizzatori circadiani sono detti zeitgeber, parola di origine tedesca che significa "che dà il tempo". Possono essere segnali di tipo luminoso o non luminoso e sono rappresentati da segnali metabolici, neuronali, alimentari, comportamentali. La luce influenza principalmente il master clock, mentre lo stato nutrizionale, ad esempio, è in grado di resettare facilmente i clocks periferici [5] (Fig. 1).

La luce percepita dalla retina è il più potente sincronizzatore del SCN, ma gli zeitgeber sono numerosissimi. La melatonina, ad esempio, è in grado di sincronizzare il meccanismo molecolare soprachiasmatico e di influenzare in maniera minore i clocks periferici. Diversi segnali ormonali mostrano altresì proprietà di sincronizzatori, ad esempio $\mathrm{i}$ glucocorticoidi, che possono modulare in maniera importante sia i clocks centrali, incluso il master clock, sia i clocks periferici. Altri zeitgeber includono ormoni che controllano l'appetito e il metabolismo energetico, come la leptina, l'insulina e la grelina. Anche i pattern comportamentali (sonno, alimentazione, attività fisica) hanno un importante ruolo nel sincronizzare, o perturbare, il sistema circadiano [5].

\section{Melatonina}

La melatonina è un ormone sintetizzato e secreto dalla ghiandola pineale, o epifisi. La sua secrezione viene regolata dalle vie afferenti noradrenergiche che giungono alla 
ghiandola pineale e controllate da impulsi soprachiasmatici. La melatonina non viene accumulata nei pinealociti, ma viene secreta subito dopo la sintesi, che vede il suo picco durante la notte sia negli animali diurni sia in quelli notturni. L'esposizione notturna a luce intensa ha un potente effetto inibitorio sulla secrezione della melatonina nell'uomo e in altri mammiferi.

I livelli plasmatici di melatonina sono regolati dal master clock attraverso le vie noradrenergiche, e il momento del picco di melatonina salivare o plasmatica (dim light melatonin onset, DLMO) viene utilizzato come marker della fase circadiana del SCN. La melatonina coopera alla sincronizzazione interna del sistema circadiano, regolando la fase degli oscillatori: un'infusione giornaliera di melatonina riesce a sincronizzare il ritmo di risposo/attività e il meccanismo molecolare soprachiasmatico in ratti mantenuti in oscurità costante [6].

La melatonina è utilizzata da anni contro i disturbi del sonno: in particolare, riduce il tempo necessario all'addormentamento e aumenta il tempo di sonno totale, con il risultato di un miglioramento generale della qualità del sonno. Si è visto che la melatonina riduce anche la latenza dell'insorgenza della fase REM [7]. L'utilizzo di melatonina esogena risulta utile in disturbi del sonno primari e secondari, in pazienti di diverse fasce di età, inclusi bambini e anziani. L'utilizzo di melatonina porta benefici anche in disturbi circadiani del sonno, essendo in grado di resettare il ritmo sonno/veglia, ad esempio nella sindrome della fase di sonno ritardata (DSPS), o in caso di ritmi sonno veglia irregolari o cicli sonno/veglia di durata diversa da 24 ore [7].

Anche disturbi del ritmo circadiano non endogeni possono trovare giovamento dall'utilizzo di melatonina: il lavoro su turni notturni e il jet-lag. Si è visto che dosi di melatonina da 0,5 e $5 \mathrm{mg}$ permettono di ridurre significativamente il jet-lag e anche che l'utilizzo di melatonina dopo un turno notturno migliora la durata e la qualità del sonno [7].

\section{Variabili metaboliche}

Tutti gli assi ormonali mostrano una ciclicità circadiana: i livelli ematici di molti ormoni seguono fluttuazioni precise, così come svariate funzioni fisiologiche, grazie agli stimoli forniti dai clocks centrali e periferici. Ad esempio, le concentrazioni plasmatiche di melatonina, cortisolo [8] e ormone della crescita $(\mathrm{GH})$ presentano specifici pattern giornalieri, così come la temperatura corporea, il volume urinario e la pressione sistolica [9]. L'effetto del sistema circadiano sull'organismo è complesso e influenza la maggior parte dei sistemi endocrini e metabolici, compresi il metabolismo energetico e quello del glucosio [10], gli assi ipotalamo-ipofisi-tiroide (HPT) e ipotalamo-ipofisi-gonade (HPG) [11], la secrezione di adipochine. Il sistema circadiano influenza, inoltre, il microbiota intestinale, che altresì presenta dei propri ritmi circadiani in termini di numerosità di popolazione e di metaboliti, ritmi a loro volta influenzabili da input comportamentali e circadiani dell'ospite.

\section{Asse ipotalamo-ipofisi-surrene (HPA): cortisolo}

I glucocorticoidi (GC) sono gli effettori steroidei dell'asse ipotalamo-ipofisi-surrene (HPA), nell'uomo e in altri mammiferi come cani e criceti, ma anche in molti pesci. Nell'uomo, il principale glucocorticoide circolante è il cortisolo, mentre in ratti, topi e rettili è il corticosterone [12]. Il cortisolo e il corticosterone vengono secreti in seguito a una complessa e articolata cascata di reazioni biochimiche che si originano dai neuroni del nucleo paraventricolare dell' $i-$ potalamo che producono l'ormone di rilascio della corticotropina (CRH o CRF), necessario per il rilascio dell'ormone adrenocorticotropo (ACTH) dall'ipofisi anteriore. Molti dei neuroni che rilasciano $\mathrm{CRH}$ producono anche il neurormone arginin-vasopressina (AVP) e costituiscono una componente integrata nel contesto dell' asse HPA, in quanto ricevono input neuronali da molte aree cerebrali. L'ACTH viene prodotto nell'ipofisi anteriore e deriva da uno specifico splicing del proormone proopiomelanocortina (POMC) ad opera di una proormone convertasi (PC). L'ACTH stimola la sintesi di cortisolo e corticosterone nella zona fascicolata della corteccia surrenale. Il cortisolo non viene accumulato in vescicole ma si diffonde fuori dalle cellule appena sintetizzato [12].

L'attività dell'asse HPA (e, quindi, della secrezione di cortisolo e corticosterone) segue 3 differenti pattern temporali. La prima modalità consiste nelle pulsazioni ultradiane (circa ogni 60 minuti), che dipendono principalmente dall'oscillazione dei livelli di ACTH a causa del feedback negativo degli stessi GC sulla secrezione di ACTH [12].

La seconda modalità di secrezione è quella circadiana, caratterizzata da un picco di corticotropina (ACTH) di ampiezza maggiore nelle prime ore del mattino. In questo modo il picco di cortisolo si manifesta nell'ora che precede il risveglio, per poi diminuire e arrivare al suo minimo la sera, tornando a crescere qualche ora dopo l'inizio della fase di riposo [8].

L'oscillazione circadiana di cortisolo deriva da una complessa regolazione nervosa ed endocrina, grazie all'azione del SCN che agisce attraverso diversi meccanismi. Esiste un collegamento con i neuroni secernenti l'ormone di rilascio della corticotropina $(\mathrm{CRH})$ nel nucleo paraventricolare (PVN) ma anche una via che coinvolge il sistema nervoso autonomo, con proiezioni neurali che innervano la corticale del surrene. Inoltre, anche i segnali alimentari e comportamentali influenzano il ritmo del cortisolo che, però, ha la tendenza ad essere molto stabile. La ritmicità del cortisolo, infatti, tende ad essere resistente ai cambiamenti acuti, come 
ad esempio l'alimentazione ad libitum, il digiuno, un sonno più lungo o più breve del solito [8].

La terza modalità di secrezione del cortisolo è quella secondaria a uno stimolo, ad esempio stress acuti o cronici. L'esposizione a stress cronici può comportare cambiamenti adattativi nell' asse HPA, con una diminuzione della risposta allo stesso tipo di stress e, di frequente, una risposta facilitata a uno stress di tipo diverso [8].

L'asse HPA influenza il sistema circadiano; infatti, i GC hanno la capacità di influenzare i clocks periferici, ma non direttamente il master clock. In ratti surrenectomizzati risulta accelerata l'inversione di fase dei clocks periferici indotta dal timing alimentare: questo fa supporre che i GC siano in grado di attenuare la desincronizzazione indotta da pasti a orari non abituali. Gli stress acuti e cronici possono alterare i segnali temporali mediati dai GC circolanti, attraverso l'alterazione della secrezione degli stessi [5].

Nel cervello, le variazioni giornaliere delle concentrazioni di GC sono in grado di sincronizzare diverse aree, come il nucleo talamico della stria terminale, del nucleo centrale dell'amigdala e dei nuclei serotoninergici del rafe [5]. Il messaggio dei GC può raggiungere il clock centrale mediante meccanismi indiretti e controllando l'espressione di geni circadiani in altre aree cerebrali che proiettano al SCN [13].

I GC possono influenzare la fase di altri ritmi ormonali, con un effetto spesso tessuto-specifico, e in molti tessuti sembrano modulare l'espressione ritmica dei geni del clock. Una buona parte del trascrittoma epatico sembra essere influenzato dall' attività della ghiandola surrenale, così come circa il $50 \%$ dei geni del tessuto muscolare risponde all'azione dei GC [13].

\section{Metabolismo circadiano del glucosio}

Come altre variabili metaboliche, anche la secrezione di insulina, l'insulino-sensibilità e le concentrazioni plasmatiche di glucosio presentano delle oscillazioni giornaliere.

È stato evidenziato un pattern circadiano della secrezione insulinica, con un picco nella prima parte della giornata e il nadir tra le 18 e mezzanotte. Questo può contribuire a spiegare la maggiore tolleranza al glucosio che si evidenzia nella prima parte della giornata, rispetto alla sera. Le oscillazioni giornaliere della tolleranza al glucosio sono in parte modulate dal ritmo dell'insulino-sensibilità generale e dal ritmo della sensibilità al glucosio delle $\beta$-cellule pancreatiche, che fungono da "sensori di glucosio" per innescare la secrezione di insulina: le $\beta$-cellule sono in grado di fornire una risposta secretoria più marcata durante il mattino [10].

La ritmicità delle concentrazioni plasmatiche di glucosio può essere in parte spiegata dalla produzione epatica dello stesso: il glucagone, che stimola la gluconeogenesi epatica, può spiegare l'oscillazione circadiana della glicemia a digiuno. Studi su roditori hanno dimostrato che anche il glucagone ha una secrezione circadiana, con picco all'inizio della fase attiva. Uno studio condotto su ratti ha mostrato come il ritmo di secrezione del glucagone fosse più evidente in ratti a digiuno rispetto a ratti normalmente nutriti, mentre in caso di lesioni al SCN la ritmicità delle concentrazioni plasmatiche di glucagone veniva a mancare. Il glucagone, così come l'insulina, risponde all'introduzione di cibo, quindi, anche in ratti con lesioni ipotalamiche, venivano mantenuti i picchi di glucagone indotti dai pasti [14].

Il sistema circadiano regola il metabolismo del glucosio mediante il master clock, che influenza processi come l'assunzione di cibo, il ritmo sonno-veglia, il dispendio energetico e la secrezione di ormoni. Il SCN contribuisce a regolare i ritmi di rilascio di cortisolo, insulina e ormone della crescita, ad esempio, che a loro volta influenzano il signalling e la secrezione dell'insulina. Studi su roditori hanno mostrato che lesioni al SCN portano a un'alterazione del ritmo della insulino-sensibilità: gli animali che avevano subito le lesioni diventavano insulino-resistenti in 8 settimane [15].

I clocks periferici svolgono un'azione altrettanto importante, attraverso una moltitudine di meccanismi: il clock intestinale, ad esempio, regola l'assorbimento di nutrienti (regolando l'espressione dei trasportatori di membrana del glucosio) e la motilità intestinale. Il clock del muscolo scheletrico permette una ciclicità dell'insulino-sensibilità di questo tessuto mediante cambiamenti nel livello e traslocazione sulla membrana del trasportatore GLUT-4, che vede un picco al mattino. Anche gli adipociti mostrano una ritmicità nell'uptake del glucosio e nella capacità ossidativa, sia nel tessuto adiposo bianco sia nel tessuto adiposo bruno. Il clock epatico è uno degli orologi periferici che risponde maggiormente ai segnali derivanti dall'assunzione di cibo; infatti, può disaccoppiarsi dal master clock se invertiamo i ritmi alimentari. L'alterazione del ritmo del clock pancreatico può causare un' alterata secrezione insulinica, in quanto CLOCK e BMAL1 attivano la secrezione di geni che regolano la produzione e il signaling dell'insulina (come InsR, Irs2), la sensibilità al glucosio (Glut2, Gck) e la crescita delle $\beta$-cellule (come i geni CyclinD1, Gsk $3 \beta, \mathrm{Hnf} 4 \alpha$ ). È stato dimostrato che la distruzione del clock pancreatico provoca alterazioni della secrezione insulinica, che possono arrivare a determinare l'insorgenza del diabete al diabete [16].

\section{Metabolismo circadiano dell'adipocita e del metabolismo energetico}

\section{Tessuto adiposo}

Il tessuto adiposo è caratterizzato da un elevato grado di eterogeneità e i diversi tipi di tessuto adiposo formano uno dei più grandi organi del corpo umano. Abbiamo varietà 
sia in termini di tipologie di depositi, sia in termini di tipologie cellulari; nel tessuto adiposo sono, infatti, presenti adipociti maturi, adipociti in fase di differenziazione, preadipociti, macrofagi, fibroblasti, strutture connettive, capillari. I depositi possono essere sottocutanei (SAT) o viscerali (VAT). Questi ultimi in particolare, se presenti in eccesso, costituiscono un forte fattore di rischio per i disordini metabolici.

Gli adipociti sono funzionalmente distinti in due tipi: adipociti bianchi e bruni, strutturalmente e funzionalmente differenti. Il tessuto adiposo bianco (WAT) rappresenta una riserva di energia a lungo termine; le sue cellule sono caratterizzate dalla presenza di un'unica grande goccia di materiale lipidico, che può occupare fino al $95 \%$ del volume cellulare. Il tessuto adiposo bruno (BAT) è più abbondante nei neonati e nell'adulto è localizzato soprattutto a livello sopraclavicolare, cervicale, interscapolare e ascellare. Le cellule del tessuto adiposo bruno sono caratterizzate da depositi lipidici multipli, da un elevato numero di mitocondri e dall'espressione della proteina disaccoppiante 1 , UCP1, che produce calore mediante il disaccoppiamento del gradiente protonico durante la respirazione mitocondriale [17].

Il tessuto adiposo bianco è considerato un organo endocrino a tutti gli effetti, in quanto esprime una grande varietà di proteine (adipochine) con funzione endocrina, ad azione sia autocrina e paracrina, sia sistemica. Tra queste, numerose citochine, tra cui leptina, adiponectina, resistina, TNF- $\alpha$, IL6 , IL-10, TGF- $\beta$, proteine coinvolte nel sistema fibrinolitico, proteine del complemento, proteine legate al metabolismo e al trasporto dei lipidi, enzimi coinvolti nel metabolismo degli steroidi, proteine del sistema renina angiotensina [17].

Inoltre, il tessuto adiposo esprime numerosi recettori che gli permettono di rispondere a segnali endocrini e centrali: sono infatti presenti recettori per insulina, glucagone, GH, angiotensina II, recettori nucleari come quello dei glucocorticoidi, ma anche recettori per citochine e catecolamine. Le funzioni endocrine e la regolazione del tessuto adiposo sono complesse e sono soggette e regolano, a loro volta, l'omeostasi energetica, il sistema immunitario, il metabolismo lipidico e del glucosio [17].

Anche il tessuto adiposo bianco contiene un orologio circadiano autonomo, sincronizzato sia dal SCN sia da segnali derivanti dall'assunzione di cibo. Nell'uomo, il $25 \%$ del trascrittoma del tessuto adiposo mostra variazioni giornaliere, con conseguenti oscillazioni nei livelli circolanti di adipochine e di molecole coinvolte nell'uptake adipocitario di glucosio e nel signaling dell'insulina, con un picco dell'insulino-sensibilità a mezzogiorno [10].

Anche il metabolismo lipidico è in parte regolato dai CLOCK e BMAL1, che influenzano l'espressione di enzimi chiave come adipose triglyceride lipase (ATGL), lipoprotein lipase (LPL) e hormone-sensitive lipase (HSL) [10]. In modo simile, il tessuto adiposo bruno mostra un ritmo gior- naliero per quanto riguarda l'uptake di glucosio: uno studio sull'uomo ha mostrato che il suo picco nel BAT avviene subito prima del risveglio [10].

Il tessuto adiposo viene modulato da numerosi segnali endocrini che, a loro volta, sono soggetti a una regolazione circadiana. Il sistema circadiano, quindi, tramite i clocks centrali e adipocitari e i segnali endocrini ritmici riesce a modulare la fisiologia del tessuto adiposo per ottimizzare l'utilizzo delle riserve energetiche. La desincronizzazione circadiana, infatti, è stata riconosciuta come fattore di rischio per l'obesità e le sue comorbidità, e l'alterazione di alcune funzioni endocrine potrebbe rappresentare un ulteriore collegamento [17].

Tra i segnali endocrini meglio caratterizzati a livello di ritmicità abbiamo i glucocorticoidi che, come output del $\mathrm{SCN}$, sono tra i fattori che permettono di sincronizzare gli oscillatori periferici al ciclo luce/buio. I glucocorticoidi hanno un effetto complesso sul tessuto adiposo che dipende, tra l'altro, dalla durata dell'esposizione e dal tipo di deposito. Ad esempio, hanno effetto lipogenico nei depositi viscerali e lipolitico in quelli sottocutanei [17]. I glucocorticoidi regolano la produzione di alcune adipochine, ad esempio aumentano l'espressione della leptina, mentre i loro effetti sull'espressione dell'adiponectina sono ancora controversi. Visto il loro ruolo antinfiammatorio, inibiscono l'espressione di citochine proinfiammatorie, come TNF- $\alpha$, IL-6, IL-8, nei macrofagi adipocitari [17].

La melatonina, ormone chiave per la sincronizzazione del sistema circadiano, è in grado di regolare diversi aspetti della fisiologia del tessuto adiposo. Studi su roditori hanno mostrato come la somministrazione cronica di melatonina portasse a una riduzione del peso corporeo e dell'adiposità in modelli animali di obesità, sia indotta dalla dieta (DIO), sia correlata all'età [17]. La melatonina regola il metabolismo energetico anche tramite il grasso bruno, incrementandone l'efficienza termogenica mediante diversi meccanismi, tra cui effetti diretti sulla biogenesi mitocondriale e l'incremento dell'azione dell'ormone tiroideo nel BAT grazie all'aumentata espressione di una specifica deiodinasi (DIO2). Melatonina esogena somministrata per via orale portava a una brownizzazione del WAT inguinale in ratti Zucker obesi [17]. Esperimenti in vitro hanno mostrato che la somministrazione ritmica di melatonina può sincronizzare la ritmicità degli adipociti in termini di funzioni metaboliche $\mathrm{e}$ secrezione delle adipochine [17].

\section{Leptina}

La leptina è un ormone anoressizzante espresso principalmente nel tessuto adiposo, ma la sua espressione è stata riportata anche nella placenta e nello stomaco. Agisce come indicatore dello stato energetico dell'organismo, sia a livello centrale sia a livello periferico per promuovere il dispendio energetico e inibire il deposito di energia. L'azione 
centrale della leptina è in opposizione a quella della grelina nel nucleo arcuato dell'ipotalamo (ARC): il suo effetto inibitorio sui neuroni NPY/AgRP e stimolante sui neuroni POMC/CART risulta in una diminuzione dell' assunzione di cibo, nella soppressione dell'appetito e nell'aumento del dispendio energetico [18].

I livelli plasmatici di leptina oscillano nell'arco delle 24 ore, con un picco all'inizio della fase di riposo, quindi nella prima fase di buio in animali diurni come l'uomo, e nella prima fase di luce negli animali notturni come ratti e topi [18]. La rimozione del SCN in roditori elimina la ritmicità della leptina, suggerendo che, anche se il ritmo di espressione della leptina dipende fortemente dall' assunzione di cibo, almeno in parte, è regolata dagli oscillatori circadiani [19]. Il ruolo della leptina nella modulazione dei clocks periferici è stato dimostrato mediante studi effettuati in topi che non producevano leptina (ob/ob) e mostravano un profilo alterato nell'espressione dei geni dei clocks nel fegato e nel WAT, mentre un alterato signalling della leptina (in topi ob/ob o db/db, con recettore mutato), causava una perturbazione nelle oscillazioni ritmiche del glucosio plasmatico [5]. Queste evidenze suggeriscono che la leptina mostri una ritmicità in risposta ai ritmi circadiani e agli input metabolici e, a sua volta, fornisca un feedback in grado di influenzare la funzione dei clocks.

In soggetti obesi, la leptina mantiene un suo ritmo, ma con picchi di minore ampiezza: questo suggerisce che una ritmicità circadiana attenuata $\mathrm{o}$ alterata potrebbe giocare un ruolo nello sviluppo di leptino resistenza e obesità [18].

\section{Adiponectina}

L'adiponectina viene espressa esclusivamente nel tessuto adiposo e i suoi livelli sierici sono inversamente correlati al grasso corporeo totale. L'adiponectina inibisce la gluconeogenesi e il rilascio di glucosio dal fegato indipendente da insulina, promuove l'insulino-sensibilità, aumenta la $\beta$ ossidazione nel muscolo scheletrico e inibisce l'accumulo di lipidi nel fegato e i processi infiammatori [17]. L'adiponectina è una proteina che forma complessi di diverse dimensioni, dai trimeri a basso peso molecolare fino ai complessi di 12-18 unità ad alto peso molecolare. Oltre alla concentrazione di questa adipochina, anche la prevalenza dei diversi tipi di complesso è importante nel mediare i suoi effetti biologici: la forma ad alto peso molecolare è la più attiva. Alcuni farmaci antidiabetici, come i tiazolidinedioni, aumentano in modo specifico la concentrazione di questo tipo di complesso [17].

I livelli di mRNA per adiponectina mostrano variazioni giornaliere nel WAT di ratti e topi, con valori maggiori la sera e nella prima parte della notte. Mentre nei ratti i livelli plasmatici di adiponectina non hanno mostrato una ritmicità rilevabile, nei topi sono stati rilevati livelli notturni maggiori di adiponectina plasmatica [20]. Nell'uomo, invece, avviene il contrario: i livelli di adiponectina raggiungono il loro picco durante il giorno [17]. Il picco mattutino dell'espressione di adiponectina può contribuire al fatto che questo momento è anche quello di massima tolleranza al glucosio, insulino-sensibilità e rimozione degli acidi grassi dal sangue [18]. Il recettore gamma attivato dai proliferatori dei perossisomi (PPAR- $\gamma$ ) partecipa sia ai meccanismi del clock, regolando positivamente l'espressione di BMAL1, sia ai meccanismi che inducono l'espressione dell'adiponectina: questo fa presupporre un collegamento tra i livelli di PPAR- $\gamma$ e i livelli di adiponectina (con conseguente aumento dell'insulino-sensibilità), più elevati durante il mattino [18].

\section{GLP-1}

Il glucagon-like peptide 1 (GLP-1) è sintetizzato sia a livello periferico, nelle cellule $\mathrm{L}$ dell'intestino e cellule $\alpha$ del pancreas, sia a livello centrale, nel nucleo del tratto solitario, nel nucleo reticolare dorsale e centrale, nel PVN, DMH e ARC, e i suoi recettori sono espressi in molte aree del sistema nervoso centrale. Il GLP-1 inibisce la secrezione di glucagone e stimola quella di insulina, in risposta a un aumento della glicemia dopo un pasto [19]. Aumenta anche il senso di sazietà sia rallentando lo svuotamento gastrico, sia agendo sui centri di regolazione della fame a livello centrale [19]. Alcuni studi hanno indagato gli effetti di un'alimentazione a tempo limitato (restricted feeding) sulla secrezione di GLP-1: in uno studio dove la finestra di alimentazione era ridotta a 4 ore, i ratti mostravano un aumento della concentrazione plasmatica di GLP-1 circa 1 ora prima del pasto, indicando che GLP-1 contribuisce, insieme ad altri fattori, all'attività anticipatoria al pasto, che consiste in un incremento dell'attività motoria nel momento della giornata che precede la disponibilità di cibo [19].

Studi su linee di cellule L umane e di ratto hanno mostrato che il GLP-1 presenta un pattern di secrezione circadiano. Le linee di cellule L del topo (mGLUTag) e umane (hNCI-H716) presentavano un ritmo cellulare autonomo nell'espressione di Bmal1, e la secrezione di GLP-1 seguiva lo stesso andamento. Inoltre, la soppressione di Bmall con palmitato nelle cellule mGLUTag è associata a un'attenuazione del rilascio di GLP-1 e, allo stesso modo, in colture intestinali primarie da topi knockout per Bmal1, la secrezione di GLP-1 risulta diminuita, anche se il meccanismo molecolare che collega l'espressione di Bmall alla secrezione dipendente dai ritmi circadiani di GLP-1 è ancora da approfondire [21].

Un interessante collegamento è stato notato tra la secrezione di GLP-1 e le proteine SNARE. Le proteine SNARE sono una famiglia di proteine di membrana che promuovono la fusione delle vescicole con le membrane cellulari, 
permettendo l'esocitosi del contenuto vescicolare. Un'alterata secrezione del GLP-1 è stata osservata sia in vitro che in vivo in cellule e modelli animali con deficit di proteine SNARE. Alcune di queste proteine, come VAMP2, SYNTAXIN1A, e SYNAPTOTAGMIN-7, hanno mostrato di giocare un ruolo essenziale nella secrezione di GLP-1 [21]. La secretagoghina (SCGN), una proteina regolatrice delle proteine SNARE, è stata indicata come potenziale collegamento tra l'espressione di Bmal1 e la secrezione di GLP-1. SCGN, infatti, è espressa nelle cellule L dell'intestino, dove mostra una ritmicità circadiana tramite la regolazione trascrizionale mediata da BMAL1. Il ritmo circadiano di GLP-1, quindi, può essere considerato dipendente dal gene del clock Bmal1 [21]. Questo potrebbe avere implicazioni per lo studio di trattamenti cronofarmacologici e le proteine SNARE potrebbero rappresentare un target per terapie contro il diabete mellito di tipo 2.

\section{Grelina}

La grelina è un ormone sintetizzato dalle cellule ossintiche dello stomaco, durante la fase di digiuno. Ha proprietà oressigene, che esplica mediante la sua azione stimolante sui neuroni NPY/AgRP del nucleo arcuato dell'ipotalamo [5]. La grelina è in grado di resettare il master clock in vitro e, se iniettata in vivo, produce un'inversione di fase dell'attività locomotoria in animali a digiuno. Roditori knock out per il gene del recettore della grelina mostrano un'attenuazione nell' attività anticipatoria al cibo. Questi dati mostrano che la grelina svolge un ruolo importante, ma non indispensabile, nell'attività anticipatoria al cibo [5].

La presenza di un clock periferico sincronizzabile mediante il cibo (food entrainable oscillator, FEO) è stata evidenziata anche nello stomaco. Le cellule ossintiche esprimono sia la grelina, sia proteine del clock come PER1 e PER2. L'espressione di PER1, di PER2 e della grelina, è ritmica se si ha un'alternanza delle condizioni di luce/buio ma resta, invece, costante in condizioni di buio e accesso al cibo ad libitum. In assenza dei geni del clock PER1 e PER2, la grelina non viene più espressa in maniera ritmica [22]. Questi risultati mostrano l'importanza dei clocks periferici come quello dello stomaco, nella regolazione dell'assunzione di cibo e della promozione dell'attività anticipatoria al pasto: alcuni tipi di obesità sono stati associati proprio a un'alterata regolazione dell'assunzione di cibo, come la sindrome da alimentazione notturna (night eating syndrome, NES) e l'iperalimentazione compulsiva [22].

\section{Patologie circadiane: la desincronizzazione}

\section{Desincronizzatori}

I perturbatori circadiani possono essere endogeni o esogeni. I polimorfismi nei geni dei clocks si associano a perturba- zione endogena del ritmo circadiano, i cui relativi aplotipi sono implicati nell'aumentato rischio di obesità. Altri perturbatori possono essere tipici di alcuni cronotipi: gli individui estremamente mattinieri o nottambuli tenderanno ad essere sempre disallineati con il ciclo luce-buio ambientale e ad avere maggiori fattori di rischio [23]. I perturbatori esogeni sono numerosi: il più potente è la luce, siamo sempre meno esposti alla luce diurna e sempre più esposti alla luce artificiale notturna, anche proveniente da schermi luminosi. L'esposizione notturna a luce intensa ha un potente effetto inibitorio sulla secrezione della melatonina nell'uomo e in altri animali [24] ed è la prima causa di desincronizzazione circadiana.

Studi cross-sectional e prospettici hanno identificato il lavoro notturno come un predittore indipendente di eccesso ponderale. Anche i pattern del sonno possono contribuire alla desincronizzazione circadiana, in quanto durante il giorno il livello di melatonina è basso e lo stato circadiano di allerta è in opposizione alla propensione al sonno, che sarà frammentario e qualitativamente peggiore [23]. Spesso il fatto stesso di posticipare la fase del sonno è accompagnato da routine non salutari che includono l'utilizzo di dispositivi luminosi e scelte alimentari dannose sia per l'orario in cui si mangia, sia per la qualità dei nutrienti scelti, ovvero cibi energeticamente densi, poiché il deficit di sonno aumenta anche l'attività delle aree cerebrali di ricompensa per il cibo [24].

Studi su animali hanno mostrato come l'alimentazione in orari non appropriati possa portare a conseguenze metaboliche negative, dal momento che i clocks periferici vanno fuori fase rispetto al master clock, che invece continua ad essere sincronizzato al ciclo luce-buio [24].

Un enorme contributo alla comprensione di tali meccanismi è stato fornito dagli studi del gruppo di Paolo Sassone Corsi, precocemente scomparso qualche mese fa. Nei topi, 10 settimane di dieta ad alto contenuto di grassi (HFD) portavano a modifiche in un grande numero di metaboliti coinvolti in diverse vie metaboliche, con particolare riferimento al metaboloma epatico, dove molti metaboliti sono espressi in maniera circadiana, controllata dai geni del clock [25]. Quando i topi vengono nutriti a orari casuali, si può avere un'inversione di fase dei clock periferici. Tale desincronizzazione circadiana influenza il controllo glicemico, perturbando la funzionalità delle $\beta$-cellule e l'insulino-sensibilità, con conseguente aumento di rischio di sviluppo di diabete di tipo 2 e malattie metaboliche [26].

È necessario, tuttavia, ricordare che, per poter estendere i risultati di studi preclinici su animali a una popolazione umana, occorre tenere in considerazione alcuni aspetti fondamentali, quali le differenze di peso, di superficie corporea, di metabolismo basale e le differenze che occorrono tra animali notturni e diurni, sia in termini di attività locomotoria sia in termini di pattern del comportamento alimentare. 
Fig. 2 Rappresentazione delle funzioni regolate dal sistema circadiano e delle relative alterazioni

\begin{tabular}{|c|c|c|c|c|}
\hline & $\begin{array}{l}\text { Sistema circadiano } \\
\text { sincronizzato }\end{array}$ & & & $\begin{array}{c}\text { Sistema circadiano } \\
\text { perturbato }\end{array}$ \\
\hline ; & $\begin{array}{l}\text { Veglia, alimentazione } \\
\text { Sonno, digiuno }\end{array}$ & SNC & $\begin{array}{l}\circ \\
\circ \\
\circ\end{array}$ & $\begin{array}{l}\text { Disturbi del sonno } \\
\text { Alterati pattern alimentari } \\
\text { Leptino resistenza }\end{array}$ \\
\hline $\begin{array}{l}* \\
* \\
0 \\
0 \\
0\end{array}$ & $\begin{array}{l}\text { Secrezione adiponectina, } \\
\text { lipogenesi } \\
\text { Secrezione leptina, lipolisi } \\
\text { Insulino sensibilità } \\
\text { Uptake del glucosio } \\
\text { Differenziazione adipocitaria }\end{array}$ & WAT & $\stackrel{\circ}{\circ}$ & $\begin{array}{l}\text { Obesità } \\
\text { Alterati pattern alimentari }\end{array}$ \\
\hline$\stackrel{\circ}{\circ}$ & $\begin{array}{l}\text { Uptake del glucosio } \\
\text { Espressione UCP1 }\end{array}$ & BAT & $\stackrel{\circ}{\circ}$ & $\begin{array}{l}\text { Iperglicemia? } \\
\text { Obesità? }\end{array}$ \\
\hline * & $\begin{array}{l}\text { Secrezione insulina, insulino } \\
\text { sensibilità delle } \beta \text {-cellule } \\
\text { Secrezione glucagone }\end{array}$ & PANCREAS & $\stackrel{\circ}{\circ}$ & $\begin{array}{l}\text { Alterata secr. insulinica } \\
\text { Iperglicemia }\end{array}$ \\
\hline ; & $\begin{array}{l}\text { Glicogenosintesi } \\
\text { Gluconeogenesi, glicogenolisi } \\
\text { Insulino sensibilità }\end{array}$ & FEGATO & $\stackrel{\circ}{\circ}$ & $\begin{array}{l}\text { Dislipidemia } \\
\text { Steatosi }\end{array}$ \\
\hline \% & $\begin{array}{l}\text { Uptake glucosio, uptake acidi } \\
\text { grassi } \\
\text { Metabolismo ossidativo } \\
\text { Insulino sensibilità }\end{array}$ & MUSC & $\stackrel{\circ}{\circ}$ & $\begin{array}{l}\text { Insulino resistenza } \\
\text { Iperglicemia }\end{array}$ \\
\hline$*$ & Secrezione grelina & STOMACO & $\circ$ & Alterazioni regolazione appetito? \\
\hline & Secrezione cortisolo & GH.SURRENALE & $\stackrel{0}{\circ}$ & $\begin{array}{l}\text { Alterazioni asse HPA } \\
\text { Desincronizzazione circadiana da } \\
\text { alterata secrezione di GC }\end{array}$ \\
\hline
\end{tabular}

\section{Patologie correlate alla desincronizzazione}

La desincronizzazione circadiana può condurre a un peggiore status metabolico e favorire molteplici patologie, che includono l'obesità, la sindrome metabolica, l'insulinoresistenza, il diabete mellito di tipo 2 , ma anche alcune forme di cancro, alterazioni del microbiota, patologie psichiatriche e neurodegenerative e, come si può facilmente intuire, disturbi del sonno (circadian rhythm sleep disorders, CRSD) (Fig. 2) [23].

\section{Alterazione dei ritmi circadiani e obesità}

Studi epidemiologici hanno evidenziato come la desincronizzazione circadiana sia una componente comune nell'obe- sità. Ad esempio, l'assunzione di cibo nella fase che normalmente dovrebbe essere dedicata al riposo influenza l'espressione dei geni del clock nel tessuto adiposo e nel fegato, aumentando la predisposizione all'obesità [27].

I disturbi del sonno sono correlati allo sviluppo di obesità e l'obesità può contribuire all'alterazione del ritmo sonno veglia, spesso a causa dell'apnea ostruttiva del sonno (OSA), ma anche per altre condizioni correlate, come disturbi gastrointestinali, asma, dolori osteoarticolari, nicturia. L'aumentata secrezione di citochine proinfiammatorie (IL-1, IL-6, TNF- $\alpha$ ) contribuisce allo sviluppo di uno stato infiammatorio cronico di basso grado. Alcune citochine mostrano una ritmicità di espressione, ad esempio IL-6 e 
TNF- $\alpha$ presentano il loro picco durante la notte, regolando, in particolare, il sonno a onde lente [27].

I risultati di molti studi evidenziano come i disturbi del sonno possano essere considerati un fattore di rischio per lo sviluppo di obesità. L'aumentata prevalenza di obesità in soggetti con sonno breve e disturbato può essere in parte spiegata dall'effetto di questo fenomeno sull'intake calorico; infatti, si assiste a un aumento del consumo di cibi grassi, ricchi di zuccheri e scarsi in fibre e micronutrienti. La perturbazione circadiana conseguente ai disturbi del sonno porta a una serie di effetti a cascata che contribuisce allo sviluppo di obesità, diabete di tipo 2 e disordini metabolici [27].

\section{Insulino-resistenza e T2DM}

Studi su roditori hanno dimostrato che lesioni del SCN portavano a un'alterazione del ritmo dell'insulino-sensibilità dell'organismo [10]. Nell'uomo, protocolli di disallineamento circadiano hanno dimostrato che esiste un controllo circadiano endogeno della tolleranza al glucosio, indipendente dalle abitudini alimentari [10].

Il master clock è responsabile della regolazione circadiana di fattori che controllano l'omeostasi energetica, come la termogenesi indotta dalla dieta, il metabolismo basale e l'attività del tessuto adiposo bruno [10]. I clocks periferici hanno un ruolo altrettanto importante, ad esempio per quanto riguarda la regolazione dell'insulino-sensibilità, della motilità intestinale e dell'assorbimento dei nutrienti. Nella $\beta$ cellula, CLOCK e BMAL1 attivano la trascrizione di geni coinvolti nella biosintesi, nel trasporto e nella secrezione glucosio-dipendente dell'insulina; in linea con questa osservazione, la perturbazione del clock pancreatico può causare una secrezione di insulina alterata [10].

L'associazione tra perturbazione circadiana e sviluppo di insulino-resistenza e diabete mellito di tipo 2 è supportata da diverse osservazioni, che includono lo sviluppo di sindrome metabolica in topi con mutazioni e polimorfismi in geni degli oscillatori circadiani (come Clock, ARNTL e Cry2), l'associazione tra l'assunzione di cibo nella fase deputata al riposo, e l'insorgenza di obesità nei topi e la diminuita tolleranza al glucosio nell'uomo in caso di desincronizzazione circadiana. Studi su modelli tessuto-specifici e su animali knockout per geni come Clock e BMAL1 hanno fornito ulteriore supporto al collegamento tra varie forme di desincronizzazione circadiana e l'insulino-resistenza [10].

Diverse mutazioni nei geni degli oscillatori circadiani possono contribuire alla suscettibilità genetica all'insorgenza di obesità, insulino-resistenza e T2DM. Studi osservazionali hanno mostrato un'associazione tra polimorfismi a singolo nucleotide in Bmal1 e il diabete mellito di tipo 2, tra specifici aplotipi del gene Clock e l'obesità e tra polimorfismi in Cry2 e un innalzamento della glicemia a digiuno [10]. In modo simile, perturbatori circadiani esogeni come l'esposizione notturna alla luce o pattern del sonno scorretti, sono stati collegati all'insorgenza di obesità e T2DM. Alcuni dei meccanismi proposti per spiegare il legame tra disturbi del sonno e l'alterazione dell'insulino-sensibilità includono un'alterazione dell' equilibrio simpato-vagale e aumentati livelli circolanti di catecolamine o cortisolo [10]. Anche le abitudini moderne e il lavoro su turni notturni, causando una discrepanza tra i ritmi comportamentali e quelli circadiani, sono associati a un maggior rischio di obesità e T2DM [10].

Sembra inoltre probabile che l'alterazione dei clocks centrali o periferici possa contribuire alla fisiopatologia dell'insulino-resistenza anche a livello tissutale, e che la desincronizzazione circadiana possa causare una mancata corrispondenza dei flussi di nutrienti. Per esempio, uno sfasamento tra la produzione epatica di glucosio, l'uptake muscolare di glucosio e l'assunzione di carboidrati con la dieta può contribuire a un innalzamento della glicemia e a un disequilibrio tra deposito e ossidazione dei lipidi e alla produzione di lipidi a livello epatico (che potrebbe contribuire all'accumulo ectopico) [10].

\section{Contromisure alla desincronizzazione circadiana}

Molti interventi differenti si sono dimostrati promettenti nell'attenuare le conseguenze metaboliche della perturbazione circadiana e del sonno, in primis la fototerapia e la regolazione dell'esposizione alla luce, il principale zeitgeber. $\mathrm{Ad}$ esempio, un trial controllato randomizzato ha mostrato come l'introduzione di condizioni di luce che simulassero la luce del sole con luci artificiali intense durante il giorno ha migliorato le funzioni cognitive, la qualità del sonno e il ritmo di attività motoria di anziani in case di riposo [28]. Il pattern del sonno può essere alterato da fattori comportamentali o esterni (come l'isolamento e il cambiamento della nostra routine quotidiana causati dall'emergenza COVID-19) e gli interventi per migliorarlo includono l'adozione di orari regolari (andare a dormire e alzarsi sempre alla stessa ora), la pratica di attività fisica, ma non nell'ultima parte della giornata, l'esposizione alla luce del sole e la riduzione dell'esposizione alla luce artificiale nelle ore serali. Altri interventi che potremmo chiamare di "medicina circadiana" includono la programmazione dei tempi di sonno, attività fisica, alimentazione, dispensazione dei farmaci (cronoterapia) [29].

Tenere in considerazione i meccanismi circadiani dei vari tessuti può portare progressi nei campi della cronoterapia e cronofarmacologia, per ottimizzare l'efficacia dei farmaci prescritti e ridurre ulteriormente gli effetti collaterali, virtualmente senza costi sanitari aggiuntivi. Nelle patologie cardiovascolari, ad esempio, esistono andamenti temporali dei fattori di rischio, o alcune condizioni sono correlate a 
pattern anomali di fattori come la pressione sanguigna (ad esempio, nell'ipertensione notturna) [29]. La cronoterapia è stata di recente indagata anche in applicazioni per la terapia del cancro [30]. Il farmaco potrà essere prescritto in funzione dei ritmi biologici endogeni, valutando le interazioni farmacodinamiche con le oscillazioni circadiane, nell'ottica di trattamenti personalizzati. Di particolare interesse risultano gli interventi sullo stile di vita, che consistono nella programmazione di alimentazione e attività fisica.

La distruzione dei ritmi circadiani e l'alimentazione in orari inappropriati possono portare a effetti negativi sulla salute metabolica: in aggiunta alle classiche considerazioni sull'apporto calorico e sulla composizione della dieta, anche un appropriato timing dei pasti può risultare utile per prevenire la desincronizzazione circadiana e i rischi metabolici. Anche la distribuzione dell'intake energetico giornaliero è da tenere in considerazione. In un trial su donne obese e sovrappeso che assumevano le stesse calorie, è emerso che quelle che mangiavano una maggiore percentuale del loro intake calorico durante le prime ore della giornata perdevano più peso di quelle che assumevano cibo soprattutto nell'ultima parte della giornata [24].

Non solo la tempistica di assunzione, ma anche la composizione degli alimenti può avere effetto sul sistema circadiano, che può essere influenzato anche dalla composizione della dieta in termini di macronutrienti, ma anche da singoli nutrienti.

Nei topi, un mangime a maggior contenuto proteico e minor contenuto in carboidrati facilita l'espressione ritmica di diversi geni del clock nel fegato e nel rene, e aumenta l'espressione media di Bmall e Cry1, rispetto a un mangime standard [24]. Nell'uomo, il passaggio da una dieta al 55\% di carboidrati e $30 \%$ di grassi a una dieta isoenergetica al $40 \%$ di carboidrati e $45 \%$ di grassi, ha ritardato e aumentato l'ampiezza del ritmo del cortisolo, ha cambiato il profilo di espressione di geni legati a processi infiammatori e metabolici e ha alterato l'espressione del gene PER nei monociti [24]. Ad esempio, tra gli acidi grassi, l'acido docosaesaenoico (DHA) influenza l'espressione di Bmal1, come è stato visto in un esperimento su una linea di cellule ipotalamiche murine. L'alcol risulta essere particolarmente distruttivo sui ritmi molecolari, endocrini e circadiani, sia nell'uomo sia in altri animali [24].

La caffeina influenza l'ampiezza e la fase dell'espressione dei geni del master clock del SCN in topi e la sua assunzione serale porta a un ritardo del ritmo circadiano nell'uomo e a un prolungamento del periodo di espressione dei geni del clock in vitro. Un' assunzione mirata di caffeina, invece, può accelerare il ripristino del ritmo circadiano a seguito di jet lag [18]. I polifenoli sono un altro gruppo di composti in grado di influenzare i ritmi circadiani a livello molecolare, ad esempio modulando l'acetilazione di Bmall e i livelli di NAD nei ratti [24].

\section{Conclusioni}

Il sistema circadiano è regolato in modo complesso e integrato e, in virtù dei suoi effetti pleiotropici sull'organismo, è facile capire come una sua alterazione possa portare a molteplici effetti negativi sulla salute. Nella società moderna siamo notevolmente esposti a una perturbazione circadiana, per le modifiche dello stile di vita occorse negli ultimi decenni: si arriva infatti a parlare di jet-lag sociale. Data la crescente evidenza del collegamento tra la perturbazione circadiana e malattie quali obesità, diabete mellito di tipo 2 e sindrome metabolica, può essere interessante un approccio integrato dal punto di vista circadiano per la cura e la prevenzione di tali patologie. La conoscenza sempre maggiore della regolazione e dell'influenza esercitata dal sistema circadiano potrebbe permettere di sviluppare trattamenti sempre più personalizzati e di ottimizzare le strategie terapeutiche, ma soprattutto preventive e di intervento sullo stile di vita.

In particolare, è importante identificare precocemente $\mathrm{i}$ disturbi del cronotipo, prima che si instauri un danno d'organo, attraverso un'attenta anamnesi alimentare e fisiologica, per poter intervenire precocemente sullo stile di vita e poter prevenire potenziali ricadute metaboliche nell'individuo.

Conflitto di interesse Gli autori Sara Benazzi, Stefania Gorini, Alessandra Feraco e Massimiliano Caprio dichiarano di non avere conflitti di interesse.

Consenso informato Lo studio presentato in questo articolo non ha richiesto sperimentazione umana.

Studi sugli animali Gli autori di questo studio non hanno eseguito studi sugli animali.

Nota della casa editrice Springer Nature rimane neutrale in riguardo alle rivendicazioni giurisdizionali nelle mappe pubblicate e nelle affiliazioni istituzionali.

\section{Bibliografia}

1. Barrea L, Pugliese G, Framondi L et al (2020) Does Sars-Cov2 threaten our dreams? Effect of quarantine on sleep quality and body mass index. J Transl Med 18(1):1-11

2. Challet E (2019) The circadian regulation of food intake. Nat Rev Endocrinol 15:393-405

3. Buhr ED, Takahashi JS (2013) Molecular components of the Mammalian circadian clock. Handb Exp Pharmacol 217:3-27

4. Hastings MH, Maywood ES, Brancaccio M (2019) The mammalian circadian timing system and the suprachiasmatic nucleus as its pacemaker. Biology 8(1):13

5. Challet E (2015) Keeping circadian time with hormones. Diabetes Obes Metab 17:76-83

6. Zawilska JB, Skene DJ, Arendt J (2009) Physiology and pharmacology of melatonin in relation to biological rhythms. Pharmacol Rep 61(3):383-410

7. Riha RL (2018) The use and misuse of exogenous melatonin in the treatment of sleep disorders. Curr Opin Pulm Med 24(6):543-548 
8. Kassi EN, Chrousos GP (2013) The central CLOCK system and the stress axis in health and disease. Hormones 12(2):172-191

9. Hastings M, O'Neill JS, Maywood ES (2007) Circadian clocks: regulators of endocrine and metabolic rhythms. J Endocrinol 195(2):187-198

10. Stenvers DJ, Scheer FA, Schrauwen P et al (2019) Circadian clocks and insulin resistance. Nat Rev Endocrinol 15(2):75-89

11. Gotlieb N, Moeller J, Kriegsfeld LJ (2018) Circadian control of neuroendocrine function: implications for health and disease. Curr Opin Physiol 5:133-140

12. Spencer RL, Deak T (2017) A users guide to HPA axis research. Physiol Behav 178:43-65

13. Kalsbeek A, Van der Spek R, Lei J et al (2012) Circadian rhythms in the hypothalamo-pituitary-adrenal (HPA) axis. Mol Cell Endocrinol 349(1):20-29

14. Ruiter M, La Fleur SE, van Heijningen C et al (2003) The daily rhythm in plasma glucagon concentrations in the rat is modulated by the biological clock and by feeding behavior. Diabetes 52(7):1709-1715

15. Coomans CP, van den Berg SA, Lucassen EA et al (2013) The suprachiasmatic nucleus controls circadian energy metabolism and hepatic insulin sensitivity. Diabetes 62(4):1102-1108

16. Marcheva B, Ramsey KM, Buhr ED et al (2010) Disruption of the clock components CLOCK and BMAL1 leads to hypoinsulinaemia and diabetes. Nature 466(7306):627-631

17. Kiehn JT, Tsang AH, Heyde I et al (2011) Circadian rhythms in adipose tissue physiology. Compr Physiol 7(2):383-427

18. Froy O, Garaulet M (2018) The circadian clock in white and brown adipose tissue: mechanistic, endocrine, and clinical aspects. Endocr Rev 39(3):261-273

19. Patton DF, Mistlberger RE (2013) Circadian adaptations to meal timing: neuroendocrine mechanisms. Front Neurosci 7:185
20. Challet E (2017) Circadian aspects of adipokine regulation in rodents. Best Pract Res Clin Endocrinol Metab 31(6):573-582

21. Konturek PC, Brzozowski T, Konturek SJ (2011) Gut clock: implication of circadian rhythms in the gastrointestinal tract. J Physiol Pharmacol 62(2):139-150

22. Biancolin AD, Martchenko A, Mitova E et al (2020) The core clock gene, Bmal1, and its downstream target, the SNARE regulatory protein secretagogin, are necessary for circadian secretion of glucagon-like peptide-1. Mol Metab 31:124-137

23. Covassin N, Singh P, Somers VK (2016) Keeping up with the clock: circadian disruption and obesity risk. Hypertension 68(5):1081-1090

24. Potter GD, Cade JE, Grant PJ, Hardie LJ (2016) Nutrition and the circadian system. Br J Nutr 116(3):434-442

25. Eckel-Mahan KL, Patel VR, De Mateo S et al (2013) Reprogramming of the circadian clock by nutritional challenge. Cell 155(7):1464-1478

26. Hawley JA, Sassone-Corsi P, Zierath JR (2020) Chrono-nutrition for the prevention and treatment of obesity and type 2 diabetes: from mice to men. Diabetologia 63(11):2253-2259

27. Muscogiuri G, Barrea L, Annunziata G et al (2019) Obesity and sleep disturbance: the chicken or the egg? Crit Rev Food Sci Nutr 59(13):2158-2165

28. Riemersma-Van Der Lek RF, Swaab DF, Twisk J et al (2008) Effect of bright light and melatonin on cognitive and noncognitive function in elderly residents of group care facilities: a randomized controlled trial. JAMA 299(22):2642-2655

29. Thosar SS, Butler MP, Shea SA (2018) Role of the circadian system in cardiovascular disease. J Clin Invest 128(6):2157-2167

30. Harper E, Talbot CJ (2019) Is it time to change radiotherapy: the dawning of chronoradiotherapy? Clin Oncol 31(5):326-335 